\title{
Molecular Features for Probing Small Amphiphilic Molecules with Self-Assembled Monolayer Protected Nanoparticles
}

\author{
Domenico Marson, ${ }^{a}$ Zbyšek Posel, ${ }^{a, b}$ and Paola Posocco ${ }^{a *}$ \\ ${ }^{a}$ Department of Engineering and Architecture, University of Trieste, Italy \\ ${ }^{b}$ Department of Informatics, Faculty of Science, Jan Evangelista Purkyně University, Ústí nad \\ Labem, Czech Republic \\ *paola.posocco@dia.units.it
}

\section{INDEX}

Additional results

Computational details

7 


\section{ADDITIONAL RESULTS}

Table S1. Summary of the structural characterization in solvent for the three SAM-AuNPs considered in this study, including: nanoparticle radius of gyration $\left(R_{g}\right)$; average number of ligand bundles; number of free chains; asphericity $(\delta)$ and relative ratio of the principal moments of inertia $\left(\left|I_{t}\right| I_{x}, \mid I_{z} / l_{y}\right)$; fraction of trans dihedrals angles. Uncertainties are reported in brackets.

\begin{tabular}{|l|c|c|c|c|c|c|c|}
\hline & $\begin{array}{c}R_{g} \\
(\mathrm{~nm})\end{array}$ & $\begin{array}{c}\text { Average } \\
\text { number of } \\
\text { bundles }^{\mathrm{a}}\end{array}$ & $\begin{array}{c}\text { Free } \\
\text { chains }^{\mathrm{b}}\end{array}$ & $\begin{array}{c}\delta^{\mathrm{c}} \\
(-)\end{array}$ & $\begin{array}{c}\left.I_{z}\right|_{x} ^{\mathrm{c}} \\
(-)\end{array}$ & $\begin{array}{c}I_{z} / l_{y}^{\mathrm{c}} \\
(-)\end{array}$ & $\begin{array}{c}\text { Fraction of } \\
\text { trans } \\
\text { dihedrals } \\
(\%)^{\mathrm{d}}\end{array}$ \\
\hline S1-AuNP & 1.50 & 2 & 9.0 & 0.86 & 3.25 & 2.34 & 89.3 \\
& $(0.01)$ & & $(4.8)$ & $(0.13)$ & $(0.35)$ & $(0.40)$ & $(2.7)$ \\
\hline S2-AuNP & 1.77 & 3 & 2.7 & 0.73 & 3.74 & 1.29 & 93.0 \\
& $(0.02)$ & & $(1.4)$ & $(0.09)$ & $(0.30)$ & $(0.13)$ & $(3.4)$ \\
\hline S3-AuNP & 1.54 & no bundles & 47.1 & 0.32 & 1.68 & 1.30 & 79.8 \\
& $(0.01)$ & & $(2.6)$ & $(0.07)$ & $(0.17)$ & $(0.12)$ & $(5.6)$ \\
\hline
\end{tabular}

a,b Ligands are assigned to the same bundle based on their relative orientation and end group distances; we used the Hierarchical Density-based Spatial Clustering of Applications with Noise

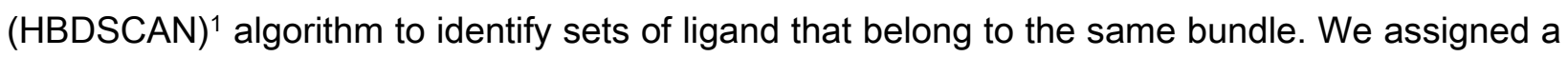
minimum number of 5 ligands to form a bundle. Chains not pertaining to a specific bundle were considered as "free". Low deviation from average values suggests that the bundling structure is rather stable on the time scale inspected (400 ns). Monolayer structures formed consistently in each system replicate.

c The asphericity $\delta$ gives an indication of shape and is defined as $I_{z}\left(I_{x}+I_{y}\right) / 2$, having defined the principal moments of the gyration tensor as $I_{z} \geq I_{y} \geq I_{x}$. Values close to 0 indicate a spherical form, while values around 1 an oblong shape (e.g., ellipsoid).

d The fraction of trans dihedrals angles $\left(-180^{\circ}<\varphi<-120^{\circ}\right.$ and $\left.120^{\circ}<\varphi<180^{\circ}\right)$ relative to the total number of dihedral angles in the ligand chain is a measure of ligand ordering. The dihedral angles were calculated taking into account all the heavy atoms of the alkyl portion and ignoring all the hydrogen atoms. A fraction of trans dihedral angles values near 1 indicates a highly ordered ligand structure and a value near 0 a highly disordered structure. 
Presence of bundles is already documented for other alkanethiols either experimentally and computationally and is related to the number of ligand carbon atoms, terminal moiety, and gold core size..$^{2-9}$ 

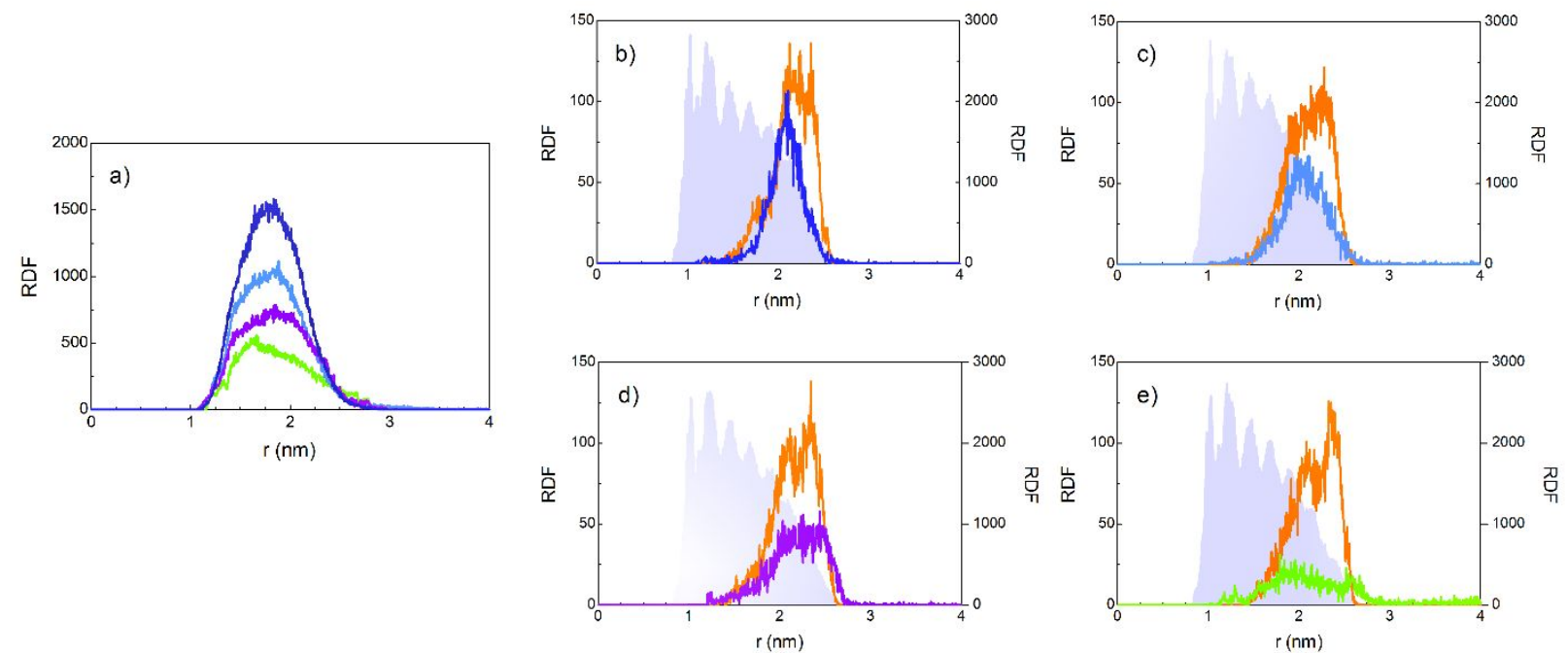

Figure S1. Distribution of A1, A2, A3 and A4 around the gold core for S1-AuNP based systems. (a) Radial distribution function (RDF) of A1 (blue), A2 (light blue), A3 (purple) and A4 (chartreuse) calculated from the center of mass of the nanoparticle. (b-e) RDF of sulfonate moieties in $\mathrm{S} 1$ ligands (orange) and amine groups in A1 (blue), A2 (light blue), A3 (purple) and A4 (chartreuse) calculated from the center of mass of the nanoparticle (left axis). For comparison the RDF of the whole $\mathbf{S 1}$ ligand is also reported as a grey area (right axis).

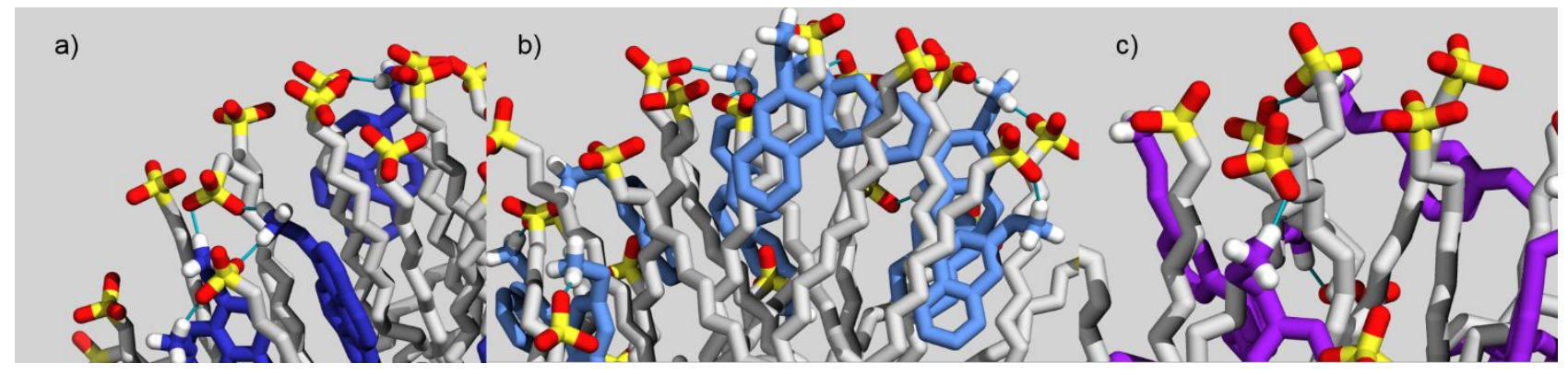


Figure S2. Selected examples of salt-bridging in A1 (a), A2 (b), and A3 (c) bound to S1-AuNP. S1 ligands are depicted as stick and colored by element. A1, A2, and A3 are represented in blue, light blue, and purple sticks, respectively. Salt-bridges are highlighted in cyan. 
Table S2. Binding free energy $\left(\Delta \mathrm{G}_{\mathrm{b}}\right)(\mathrm{kcal} / \mathrm{mol})$ and components calculated with MM/PBSA approach and binding constant $(\mathrm{K})\left(\mathrm{M}^{-1}\right)$ taken from Gabrielli et al. ${ }^{10}$ of analytes $\mathrm{A} 1, \mathrm{~A} 2, \mathrm{~A} 3, \mathrm{~A} 4$ to S1-AuNP and S3-AuNP at room temperature.

\begin{tabular}{|c|c|c|c|c|c|c|}
\hline System & $\Delta \mathrm{H}_{\text {nonpol }}$ & $\Delta \mathrm{H}_{\text {pol }}$ & $\Delta \mathrm{H}$ & $-\mathrm{T} \Delta \mathrm{S}$ & $\Delta \mathrm{G}_{\mathrm{b}}$ & $\mathrm{K}$ \\
\hline S1-AuNP/A1 & $-53.2 \pm 0.3$ & $-9.1 \pm 1.6$ & $-62.3 \pm 1.6$ & $5.0 \pm 1.0$ & $-57.3 \pm 1.9$ & $(2.2 \pm 0.1) \times 10^{6}$ \\
\hline S1-AuNP/A2 & $-32.1 \pm 0.5$ & $-8.6 \pm 1.1$ & $-40.7 \pm 1.2$ & $3.2 \pm 1.1$ & $-37.5 \pm 1.6$ & $(6.1 \pm 1.4) \times 10^{5}$ \\
\hline S1-AuNP/A3 & $-28.4 \pm 0.4$ & $-5.8 \pm 1.2$ & $-34.2 \pm 1.3$ & $1.9 \pm 1.0$ & $-32.3 \pm 1.6$ & $(4.1 \pm 1.2) \times 10^{5}$ \\
\hline S1-AuNP/A4 & $-7.9 \pm 0.4$ & $4.0 \pm 0.9$ & $-3.9 \pm 1.04$ & $0.9 \pm 0.6$ & $-3.0 \pm 1.2$ & $\begin{array}{c}\text { no binding } \\
\text { observed }\end{array}$ \\
\hline S3-AuNP/A1 & $-31.2 \pm 0.2$ & $-17.5 \pm 1.1$ & $-48.7 \pm 1.1$ & $8.3 \pm 1.2$ & $-40.4 \pm 1.6$ & $(1.3 \pm 0.1) \times 10^{6}$ \\
\hline S3-AuNP/A2 & $-25.3 \pm 0.2$ & $-12.1 \pm 0.8$ & $-37.4 \pm 0.9$ & $7.0 \pm 1.0$ & $-30.4 \pm 1.3$ & $(5.1 \pm 0.3) \times 10^{5}$ \\
\hline S3-AuNP/A3 & $-18.9 \pm 0.3$ & $-8.8 \pm 1.1$ & $-27.7 \pm 1.1$ & $3.3 \pm 0.9$ & $-24.4 \pm 1.4$ & $(9.3 \pm 1.2) \times 10^{4}$ \\
\hline S3-AuNP/A4 & $-5.8 \pm 0.3$ & $3.3 \pm 0.9$ & $-2.5 \pm 1.0$ & $1.3 \pm 0.6$ & $-1.2 \pm 1.1$ & - \\
\hline
\end{tabular}

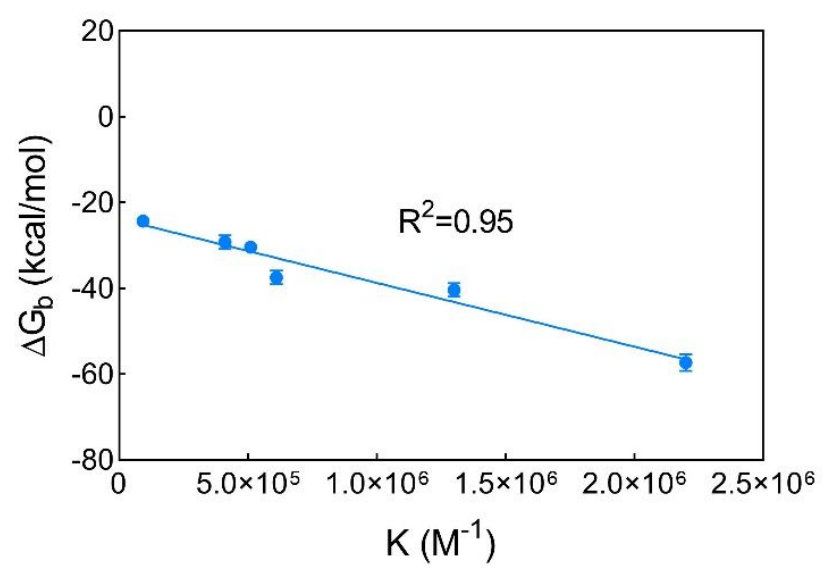

Figure S3. Correlation between free energy of binding $\left(\Delta G_{b}\right)$ and affinity constant $(K)$ for systems in Table S2. 

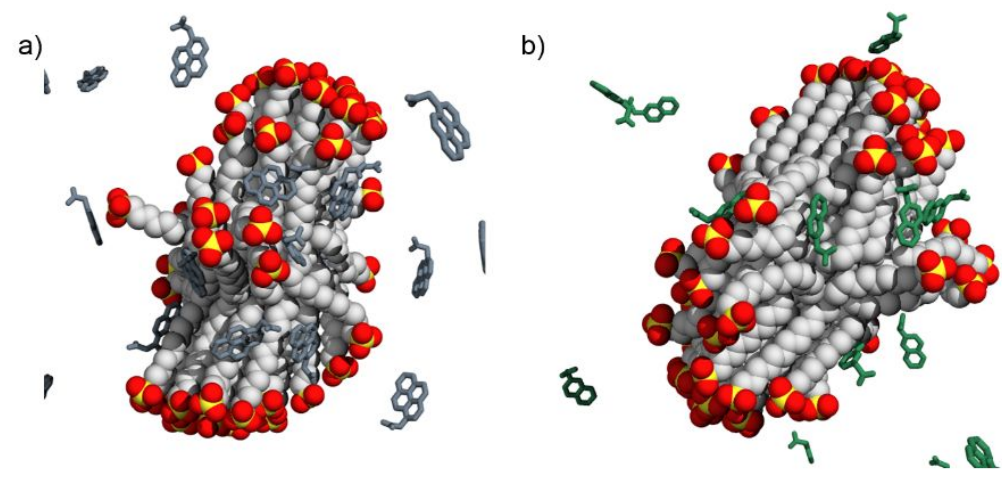

Figure S4. MD snapshots of AN1 (a) and AN2 (b) with S1-AuNP. S1 ligands are depicted as spheres and colored by element. AN1 and AN2 are represented in grey and green sticks, respectively. Water and ions are not shown for the sake of clarity.

a)

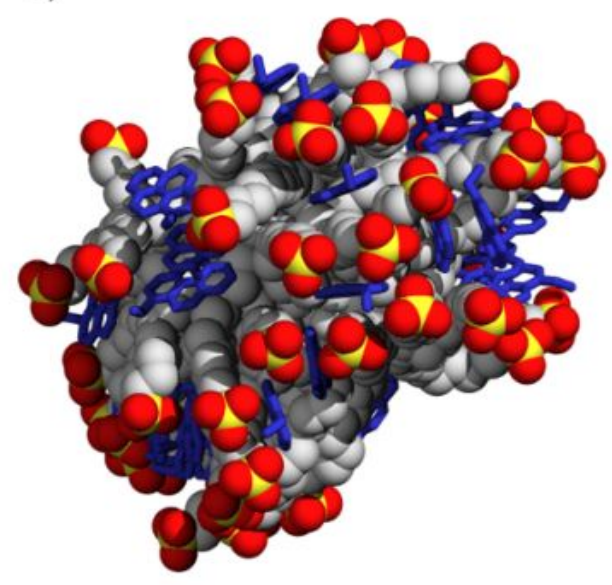

b)

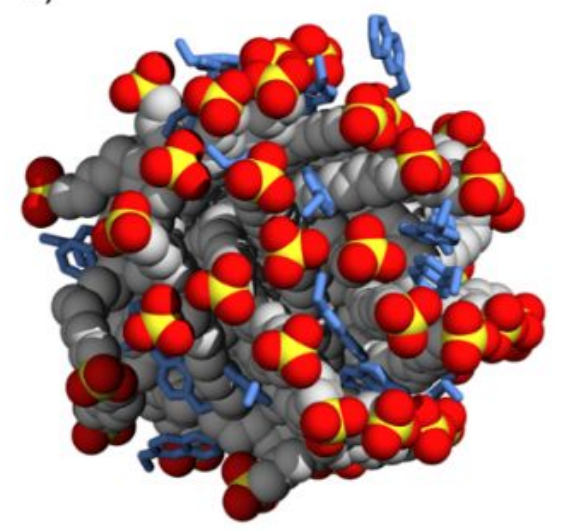

Figure S5. Top view showing interligand placement of $A 1$ (a) and A2 (b) when bound to S1-AuNP. S1 ligands are depicted as spheres and colored by element. A1 and A2 are represented in blue and light blue sticks, respectively. Water and ions are not shown for the sake of clarity. 
a)

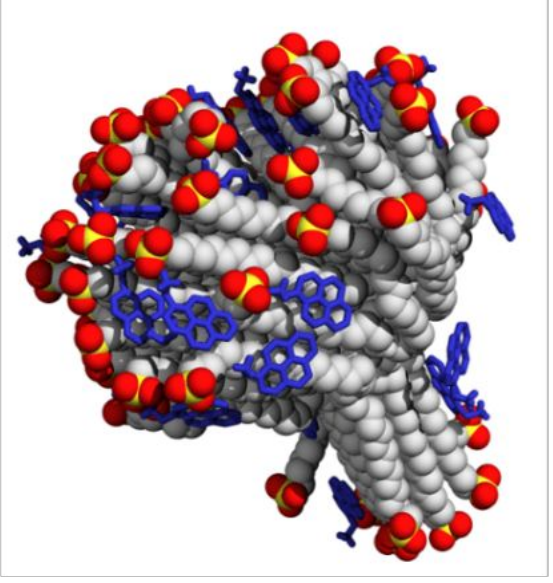

b)

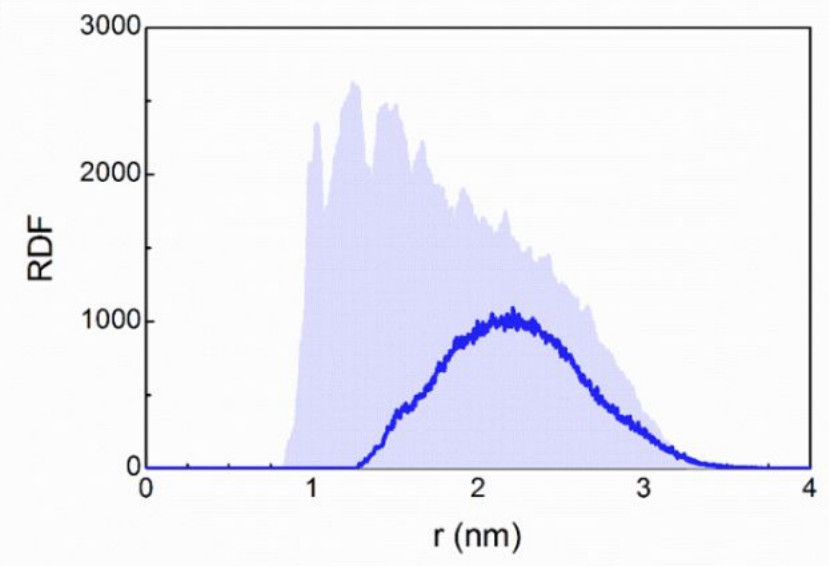

Figure S6. (a) MD snapshots of A1 with S2-AuNP. S2 ligands are depicted as spheres and colored by element. A1 is represented in blue sticks. Water and ions are not shown for the sake of clarity. (b) Radial distribution function (RDF) of S3 ligands (grey) and A1 (blue) calculated from the center of mass of the nanoparticle. 
Table S3. Structural characterization of S2-AuNP upon binding of A1, including: nanoparticle radius of gyration $\left(R_{g}\right)$; average number of ligand bundles; number of free chains; asphericity $(\delta)$ and relative ratio of the principal moments of inertia $\left(I_{z} / l_{x}, l_{z} / l_{y}\right)$; fraction of trans dihedrals angles. Uncertainties are reported in brackets.

\begin{tabular}{|l|c|c|c|c|c|c|c|}
\hline & $\begin{array}{c}R_{g} \\
(\mathrm{~nm})\end{array}$ & $\begin{array}{c}\text { Average } \\
\text { number of } \\
\text { bundles }\end{array}$ & $\begin{array}{c}\text { Free } \\
\text { chains }\end{array}$ & $\begin{array}{c}\delta \\
(-)\end{array}$ & $\begin{array}{c}I_{2} / I_{x} \\
(-)\end{array}$ & $\begin{array}{c}I_{2} / l_{y} \\
(-)\end{array}$ & $\begin{array}{c}\text { Fraction of } \\
\text { trans } \\
\text { dihedrals (\%) }\end{array}$ \\
\hline $\mathrm{A} 1$ & 1.78 & 2 & 1.8 & 0.81 & 3.61 & 1.43 & 91.9 \\
& $(0.01)$ & & $(1.1)$ & $(0.11)$ & $(0.26)$ & $(0.17)$ & $(3.3)$ \\
\hline
\end{tabular}

Table S4. Structural characterization of S1-AuNP upon A1, A2, A3 and A4 binding, including: nanoparticle radius of gyration $\left(R_{g}\right)$; average number of ligand bundles; number of free chains; asphericity $(\delta)$ and relative ratio of the principal moments of inertia $\left(I_{z} / I_{x}, I_{z} / I_{y}\right)$; fraction of trans dihedrals angles. Uncertainties are reported in brackets.

\begin{tabular}{|l|c|c|c|c|c|c|c|}
\hline & $\begin{array}{c}R_{g} \\
(\mathrm{~nm})\end{array}$ & $\begin{array}{c}\text { Average } \\
\text { number of } \\
\text { bundles }\end{array}$ & $\begin{array}{c}\text { Free } \\
\text { chains }\end{array}$ & $\begin{array}{c}\delta \\
(-)\end{array}$ & $\begin{array}{c}I_{2} / I_{x} \\
(-)\end{array}$ & $\begin{array}{c}I_{2} \\
(-)\end{array}$ & $\begin{array}{c}\text { Fraction of } \\
\text { trans } \\
\text { dihedrals (\%) }\end{array}$ \\
\hline A1 & 1.56 & 2 & 4.4 & 0.58 & 2.31 & 1.70 & 88.8 \\
& $(0.01)$ & & $(2.9)$ & $(0.12)$ & $(0.22)$ & $(0.29)$ & $(2.7)$ \\
\hline A2 & 1.57 & 2 & 3.3 & 0.65 & 2.48 & 1.88 & 89.2 \\
& $(0.01)$ & & $(2.3)$ & $(0.12)$ & $(0.26)$ & $(0.29)$ & $(2.5)$ \\
\hline A3 & 1.55 & 2 & 9.9 & 0.90 & 3.08 & 2.51 & 85.6 \\
& $(0.01)$ & & $(8.2)$ & $(0.09)$ & $(0.26)$ & $(0.27)$ & $(2.7)$ \\
\hline A4 & 1.54 & 2 & 9.8 & 0.82 & 3.11 & 2.13 & 89.7 \\
& $(0.02)$ & & $(5.3)$ & $(0.09)$ & $(0.31)$ & $(0.26)$ & $(2.6)$ \\
\hline
\end{tabular}

Table S5. Number of contact molecules $\left(N_{c}\right)$ and total number of ion pairs and water bridges $\left(N_{h}\right)$ for $S 1$ and S3-AuNP in complex with A1, A2, A3, and A4.

\begin{tabular}{|l|c|c|c|c|c|c|c|c|}
\hline \multirow{2}{*}{} & \multicolumn{2}{|c|}{ A1 } & \multicolumn{2}{c|}{ A2 } & \multicolumn{2}{c|}{ A3 } & \multicolumn{2}{c|}{ A4 } \\
\cline { 2 - 8 } & $\mathrm{N}_{\mathrm{c}}$ & $\mathrm{N}_{\mathrm{h}}$ & $\mathrm{N}_{\mathrm{c}}$ & $\mathrm{N}_{\mathrm{h}}$ & $\mathrm{N}_{\mathrm{c}}$ & $\mathrm{N}_{\mathrm{h}}$ & $\mathrm{N}_{\mathrm{c}}$ & $\mathrm{N}_{\mathrm{h}}$ \\
\hline $\begin{array}{l}\text { S1- } \\
\text { AuNP }\end{array}$ & $30 \pm 0$ & $27 \pm 4$ & $29 \pm 1$ & $26 \pm 5$ & $27 \pm 2$ & $26 \pm 4$ & $19 \pm 3$ & $16 \pm 4$ \\
\hline $\begin{array}{l}\text { S3- } \\
\text { AuNP }\end{array}$ & $30 \pm 0$ & $27 \pm 4$ & $29 \pm 1$ & $28 \pm 2$ & $29 \pm 1$ & $27 \pm 3$ & $17 \pm 3$ & $21 \pm 5$ \\
\hline
\end{tabular}


Table S6. Structural characterization of S3-AuNPs upon A1, A2, A3 and A4 binding, including: nanoparticle radius of gyration $\left(R_{g}\right)$; average number of ligand bundles; number of free chains; asphericity $(\delta)$ and relative ratio of the principal moments of inertia $\left(I_{z} / l_{x}, I_{z} / l_{y}\right)$; fraction of trans dihedrals angles. Uncertainties are reported in brackets.

\begin{tabular}{|l|c|c|c|c|c|c|c|}
\hline & $\begin{array}{c}R_{g} \\
(\mathrm{~nm})\end{array}$ & $\begin{array}{c}\text { Average } \\
\text { number of } \\
\text { bundles }\end{array}$ & $\begin{array}{c}\text { Free } \\
\text { chains }\end{array}$ & $\begin{array}{c}\delta \\
(-)\end{array}$ & $\begin{array}{c}I_{\boldsymbol{Z}} / I_{x} \\
(-)\end{array}$ & $\begin{array}{c}I_{\boldsymbol{Z}} / I_{y} \\
(-)\end{array}$ & $\begin{array}{c}\text { Fraction of } \\
\text { trans } \\
\text { dihedrals (\%) }\end{array}$ \\
\hline A1 & 1.56 & no bundles & 45.2 & 0.33 & 1.64 & 1.35 & 81.3 \\
& $(0.01)$ & & $(2.7)$ & $(0.06)$ & $(0.11)$ & $(0.10)$ & $(7.7)$ \\
\hline A2 & 1.56 & no bundles & 48.1 & 0.31 & 1.69 & 1.25 & 79.7 \\
& $(0.02)$ & & $(1.9)$ & $(0.07)$ & $(0.17)$ & $(0.12)$ & $(8.7)$ \\
\hline A3 & 1.54 & no bundles & 46.4 & 0.27 & 1.57 & 1.24 & 81.0 \\
& $(0.01)$ & & $(2.5)$ & $(0.07)$ & $(0.15)$ & $(0.12)$ & $(6.3)$ \\
\hline A4 & 1.56 & no bundles & 47.0 & 0.35 & 1.89 & 1.25 & 80.3 \\
& $(0.02)$ & & $(2.2)$ & $(0.10)$ & $(0.29)$ & $(0.15)$ & $(6.9)$ \\
\hline
\end{tabular}
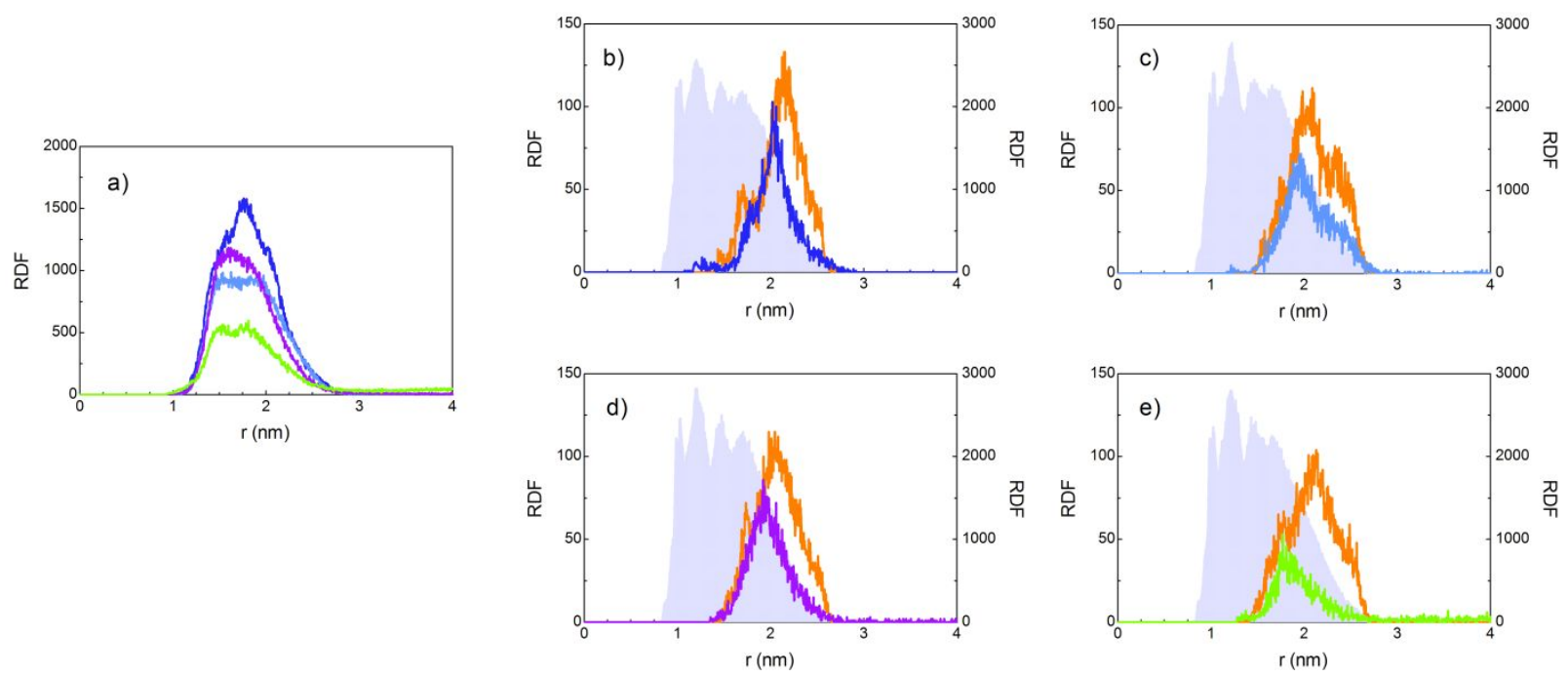

Figure S7. Distribution of A1, A2, A3 and A4 around the gold core for S3-AuNP based systems. (a) Radial distribution function (RDF) of A1 (blue), A2 (light blue), A3 (purple) and A4 (chartreuse) calculated from the center of mass of the nanoparticle. (b-e) RDF of sulfonate moieties in S3 ligands (orange) and amine groups in A1 (blue), A2 (light blue), A3 (purple) and A4 (chartreuse) calculated from the center of mass of the nanoparticle (left axis). For comparison the RDF of the whole $\mathbf{S 3}$ ligand is also reported as a grey area (right axis). 


\section{COMPUTATIONAL DETAILS}

\section{Molecular mechanics Poisson-Boltzmann surface area (MM/PBSA) methodology}

Binding free energies were computed applying the MM/PBSA methodology. Details on the methodology were reviewed recently in [11,12] and are summarized here in the following paragraphs. According to the MM/PBSA approach, the free energy of binding an analyte (A) to a nanoparticle $(\mathrm{N})$ to form a complex $(\mathrm{NA})$ in solution $\left(\Delta G_{b, \text { solv }}=\Delta G_{b}\right)$

$\Delta G_{b, \text { solv }}=\Delta G_{N A, \text { solv }}-\left(\Delta G_{N, \text { solv }}+\Delta G_{A, s o l v}\right)$

can be expressed as

$\Delta \mathrm{G}_{b, \text { solv }}=\Delta H-T \Delta S=\left\langle\Delta E_{M M}\right\rangle+\left\langle\Delta G_{\text {sol }}\right\rangle-T\left\langle\Delta S_{\text {solute }}\right\rangle$

$\Delta E_{M M}$ represents the molecular mechanical energy change in gas phase, $\Delta G_{\text {solv }}$ the solvation free energy change, and $-\mathrm{T} \Delta S_{\text {solute }}(=-\mathrm{T} \Delta S)$ the conformational entropy change of the solute upon binding.

$\Delta E_{M M}$ includes three terms:

$\Delta E_{M M}=\Delta E_{\text {int }}+\Delta E_{\text {ele }}+\Delta E_{v d W}$

the changes in the internal energies $\Delta E_{\text {int }}$ (bond, angle, and dihedral energies), the electrostatic energy change $\Delta E_{e l e}$, and the van der Waals energy change $\Delta E_{v a W}$.

$\Delta G_{s o / v}$ is separated into electrostatic solvation energy $\Delta G_{p_{-} s o l v}$ (polar contribution) and nonpolar contribution $\Delta G_{n p_{-} s o l v}$ between the solute and the continuum solvent. The polar contribution $\Delta G_{p_{-} s o l v}$ was calculated using an implicit solvent model, here the Poisson-Boltzmann (PB) model ${ }^{12}$. The nonpolar solvation energy arises from the solute cavity formation within the solvent and van der Waals interactions between the solute and the solvent around the cavity and is estimated using a fast LCPO algorithm. ${ }^{13}$ The surface tension ( $\mathrm{Y}$ ) and correction term (b) were set to 0.00542 $\mathrm{kcal}^{*} \mathrm{~mol}^{-1 *} \AA^{-2}$ and $0.92 \mathrm{kcal}^{*} \mathrm{~mol}^{-1}$ values, respectively. The change in conformational entropy - $T \Delta S_{\text {solute }}$ was computed by normal-mode analysis as implemented in MM-PBSA.py and nmode programs of AmberTools18. For free energy calculation, a solute dielectric constant $\varepsilon_{\text {in }}=2$ was employed to account for the hydrophobic nature of the inner portion of the monolayer.

Angle brackets in equation (2) indicate averages from an ensemble of representative structures, obtained extracting relevant frames from an equilibrated molecular dynamics trajectory. The 
computation for the non-entropic terms was averaged over 100 configurations evenly extracted from data collection trajectories, while the entropic term was averaged over a subset of 10 configurations (out of 100) for each independent simulation. Three replicates were considered for each system.

To account for binding-induced conformational changes, we applied a "multi-trajectory"14 approach taking three trajectories from three independent simulations (the nanoparticle in solution, the analytes in solution, and the nanoparticle-analytes complex in solution) to compute the different terms in equation (1). 


\section{REFERENCES}

1. (a) Campello, R. J. G. B.; Moulavi, D.; Sander, J. In Density-Based Clustering Based on Hierarchical Density Estimates, Berlin, Heidelberg, Springer Berlin Heidelberg: Berlin, Heidelberg, 2013; pp 160-172; (b) Mclnnes, L.; Healy, J. In Accelerated Hierarchical Density Based Clustering, 2017 IEEE International Conference on Data Mining Workshops (ICDMW), 18-21 Nov. 2017; 2017; pp 33-42.

2. Heikkilä, E.; Gurtovenko, A. A.; Martinez-Seara, H.; Häkkinen, H.; Vattulainen, I.; Akola, J., Atomistic Simulations of Functional Au144(SR)60 Gold Nanoparticles in Aqueous Environment. J. Phys. Chem. C 2012, 116, 9805-9815.

3. Bolintineanu, D. S.; Lane, J. M. D.; Grest, G. S., Effects of Functional Groups and Ionization on the Structure of Alkanethiol-Coated Gold Nanoparticles. Langmuir 2014, 30, 1107511085.

4. Koch, A. H. R.; Lévêque, G.; Harms, S.; Jaskiewicz, K.; Bernhardt, M.; Henkel, A.; Sönnichsen, C.; Landfester, K.; Fytas, G., Surface Asymmetry of Coated Spherical Nanoparticles. Nano Lett. 2014, 14, 4138-4144.

5. Giri, A. K.; Spohr, E., Conformational Equilibria of Organic Adsorbates on Nanostructures in Aqueous Solution: MD Simulations. J. Phys. Chem. C 2015, 119, 25566-25575.

6. Giri, A. K.; Spohr, E., Influence of Chain Length and Branching on the Structure of Functionalized Gold Nanoparticles. J. Phys. Chem. C 2018, 122, 26739-26747.

7. Chew, A. K.; Van Lehn, R. C., Effect of Core Morphology on the Structural Asymmetry of Alkanethiol Monolayer-Protected Gold Nanoparticles. J. Phys. Chem. C 2018, 122, 2628826297.

8. Kister, T.; Monego, D.; Mulvaney, P.; Widmer-Cooper, A.; Kraus, T., Colloidal Stability of Apolar Nanoparticles: The Role of Particle Size and Ligand Shell Structure. ACS Nano 2018, 12, 5969-5977.

9. Wu, M.; Vartanian, A. M.; Chong, G.; Pandiakumar, A. K.; Hamers, R. J.; Hernandez, R.; Murphy, C. J., Solution NMR Analysis of Ligand Environment in Quaternary AmmoniumTerminated Self-Assembled Monolayers on Gold Nanoparticles: The Effect of Surface Curvature and Ligand Structure. J. Am. Chem. Soc. 2019, 141, 4316-4327.

10. Gabrielli, L.; Rosa-Gastaldo, D.; Salvia, M.-V.; Springhetti, S.; Rastrelli, F.; Mancin, F., Detection and Identification of Designer Drugs by Nanoparticle-based NMR Chemosensing. Chem. Sci. 2018, 9, 4777-4784. 
11. Wang, E.; Sun, H.; Wang, J.; Wang, Z.; Liu, H.; Zhang, J. Z. H.; Hou, T., End-Point Binding Free Energy Calculation with MM/PBSA and MM/GBSA: Strategies and Applications in Drug Design. Chem. Rev. 2019, 119, 9478-9508.

12. Wang, C.; Greene, D. A.; Xiao, L.; Qi, R.; Luo, R., Recent Developments and Applications of the MMPBSA Method. Front. Mol. Biosci. 2018, 4, 87.

13. Homeyer, N.; Gohlke, H., Free Energy Calculations by the Molecular Mechanics Poisson-Boltzmann Surface Area Method. Mol. Inform. 2012, 31, 114-122.

14. Elder, R. M.; Jayaraman, A., Molecular Simulations of Polycation-DNA Binding Exploring the Effect of Peptide Chemistry and Sequence in Nuclear Localization Sequence Based Polycations. J. Phys. Chem. B 2013, 117, 11988-11999. 